\title{
Priming With Intermittent Theta Burst Transcranial Magnetic Stimulation Promotes Spinal Plasticity Induced by Peripheral Patterned Electrical Stimulation
}

\author{
Tomofumi Yamaguchi 1,2,3,4 Toshiyuki Fujiwara ${ }^{5}$, Su-Chuan Lin ${ }^{6}$, Yoko Takahashi², \\ Kozo Hatori ${ }^{5}$, Meigen Liu $^{2}$ and Ying-Zu Huang ${ }^{6,7 *}$
}

\begin{abstract}
${ }^{1}$ Department of Physical Therapy, Yamagata Prefectural University of Health Sciences, Yamagata, Japan, ${ }^{2}$ Department of Rehabilitation Medicine, Keio University School of Medicine, Keio University, Tokyo, Japan, ${ }^{3}$ Postdoctoral Fellow for Research Abroad (JSPS), Tokyo, Japan, ${ }^{4}$ Department of Neuroscience, University of Copenhagen, Copenhagen, Denmark, ${ }^{5}$ Department of Rehabilitation Medicine, Juntendo University Graduate School of Medicine, Juntendo University, Tokyo, Japan, ${ }^{6}$ Neuroscience Research Center and Department of Neurology, Chang Gung Memorial Hospital and Chang Gung University College of Medicine, Taoyuan, Taiwan, ${ }^{7}$ Institute of Cognitive Neuroscience, National Central University, Taoyuan, Taiwan
\end{abstract}

\section{OPEN ACCESS}

Edited by: Antonio Oliviero,

Fundación del Hospital Nacional

de Parapléjicos, Spain

Reviewed by:

Casto Rivadulla,

University of A Coruña, Spain

Claudia Ammann,

Centro Integral en Neurociencias HM CINAC, Spain

*Correspondence: Ying-Zu Huang yzhuang@cgmh.org.tw

Specialty section: This article was submitted to

Neurodegeneration,

a section of the journal Frontiers in Neuroscience

Received: 10 January 2018 Accepted: 05 July 2018 Published: 24 July 2018

Citation:

Yamaguchi T, Fujiwara T, Lin S-C,

Takahashi Y, Hatori K, Liu M and

Huang Y-Z (2018) Priming With

Intermittent Theta Burst Transcranial Magnetic Stimulation Promotes Spinal Plasticity Induced by Peripheral Patterned Electrical Stimulation. Front. Neurosci. 12:508. doi: 10.3389/fnins.2018.00508
This study explored the effect of corticospinal activity on spinal plasticity by examining the interactions between intermittent theta burst transcranial magnetic stimulation (iTBS) of the motor cortex and peripheral patterned electrical stimulation (PES) of the common peroneal nerve (CPN). Healthy volunteers $(n=10)$ received iTBS to the tibialis anterior (TA) muscle zone of the motor cortex and PES of the CPN in three separate sessions: (1) iTBS-before-PES, (2) iTBS-after-PES, and (3) sham iTBS-before-PES. The PES protocol used $10100-\mathrm{Hz}$ pulses every $2 \mathrm{~s}$ for $20 \mathrm{~min}$. Reciprocal inhibition (RI) from the TA to soleus muscle and motor cortical excitability of the TA and soleus muscles were assessed at baseline, before PES, and 0, 15, 30, and 45 min after PES. When compared to the other protocols, iTBS-before-PES significantly increased changes in disynaptic RI for 15 min and altered long-loop presynaptic inhibition immediately after PES. Moreover, the iTBS-induced cortical excitability changes in the TA before PES were correlated with the enhancement of disynaptic RI immediately after PES. These results demonstrate that spinal plasticity can be modified by altering cortical excitability. This study provides insight into the interactions between modulation of corticospinal excitability and spinal $\mathrm{RI}$, which may help in developing new rehabilitation strategies.

Keywords: H-reflex, disynaptic reciprocal inhibition, presynaptic inhibition, spinal plasticity, non-invasive brain stimulation

\section{INTRODUCTION}

Synaptic plasticity in the spinal cord plays an important role in functional recovery of the lower extremities and in recovery of walking function after spinal cord injury (SCI) and stroke (Wolpaw and Tennissen, 2001; Wolpaw, 2007; Wang et al., 2012; Howlett et al., 2015; Yamaguchi et al., 2016). Therefore, a rehabilitative strategy for enhancing spinal plasticity could be beneficial for patients with central nervous system lesions. 
One strategy for the enhancement of spinal plasticity is peripheral patterned electrical stimulation (PES), which enhances spinal reciprocal inhibition (RI) (Perez et al., 2003). However, in patients with incomplete SCI the plasticity induced by PES is only observed immediately after stimulation (Yamaguchi et al., 2016), and in healthy individuals it only lasts $10 \mathrm{~min}$ at most (Perez et al., 2003; Fujiwara et al., 2011; Yamaguchi et al., 2016; Takahashi et al., 2017). The short duration of the effects of PES limits its clinical applicability for rehabilitation. Therefore, new strategies to enhance spinal plasticity are needed.

There are indications that supraspinal modulation may strengthen the plastic changes in spinal circuits that are induced by PES (Chen et al., 2006; Fujiwara et al., 2011; Yamaguchi et al., 2013, 2016; Takahashi et al., 2017). Transcranial direct current stimulation (tDCS) is a non-invasive brain stimulation technique that can alter motor cortex excitability (Nitsche and Paulus, 2000). The spinal plasticity induced by PES can be modulated by anodal and cathodal tDCS (Fujiwara et al., 2011). Yamaguchi et al. (2016) reported that anodal tDCS of the motor cortex extended subsequent PES-induced plastic changes in spinal RI to at least $20 \mathrm{~min}$, even in patients with incomplete SCI. These results suggest that combining anodal tDCS and PES may be a useful strategy for enhancing spinal plasticity. However, the cited studies did not examine changes in motor cortical excitability, so the relationship between motor cortical excitability and PES-induced spinal plasticity remains unclear.

The time sequence of cortical stimulation and sensory inputs may also be important. Many studies have reported effects of stimulus timing on the efficacy of interventions capable of inducing plasticity (Iyer et al., 2003; Ridding and Ziemann, 2010; Müller-Dahlhaus and Ziemann, 2015). Paired associative stimulation (PAS), which induces plasticity in the motor cortex, was reported to alter the subsequent learning effect of motor practice in a way that was dependent on the interval between PAS and motor learning (Jung and Ziemann, 2009). In addition, voluntary muscle contraction is known to influence the effects of theta burst stimulation (TBS) on brain plasticity, and the temporal relationship between muscle contraction and TBS (i.e., contraction before, during, or after TBS) may affect the direction of influence, as it can enhance, abolish, or reverse the effects of TBS (Gentner et al., 2008; Huang et al., 2008). These results suggest that the temporal relationship between cortical stimulation and PES could affect plasticity in both the spinal cord and brain.

Intermittent theta burst stimulation (iTBS) is a repetitive transcranial magnetic stimulation (rTMS) protocol that uses a short stimulation period (190 s). iTBS alters motor cortex excitability comparably to other rTMS and tDCS protocols that involve longer stimulation (10 or $20 \mathrm{~min}$ ) (Huang et al., 2005; Jeffery et al., 2007; McAllister et al., 2009; Goldsworthy et al., 2012; Tatemoto et al., 2013), and is also capable of enhancing motor cortical excitability evoked by stimulation of the cortical lower limb area (Jeffery et al., 2007; Tatemoto et al., 2013; Giboin et al., 2016). These observations suggest that iTBS might be usable to modify PES-induced plasticity, and furthermore the brief stimulation used in this protocol makes it more suitable than other protocols for examining the influences of the timing and magnitude of motor cortical excitability changes on the spinal plasticity induced by PES.

In the present study we applied iTBS to modulate motor cortical excitability, and examined its time-dependent effects on the enhancement of spinal RI induced by PES in healthy individuals. In addition, we tested the effects of iTBS delivered before and after PES on motor cortical excitability by using TMS-elicited motor-evoked potentials (MEP) to elucidate the correlation between changes in motor cortical excitability and spinal plasticity.

\section{MATERIALS AND METHODS}

\section{Participants}

Ten healthy subjects who responded positively to TBS in previous experiments [eight women and two men, aged 33-58 years \{mean \pm standard deviation (SD), $42.8 \pm 8.3$ years\}] were recruited from our bank of volunteers. Previous investigations have demonstrated that healthy individuals show relatively consistent responses to repeated iTBS sessions (Hinder et al., 2014). None of the participants had a history of neurological disease or were receiving any medication affecting the central nervous system. All participants provided written informed consent prior to participation. The study was approved by the Institutional Review Board of Chang Gung Memorial Hospital in Taiwan and conformed to the tenets of the Declaration of Helsinki.

\section{Electromyography}

The participants were seated in a comfortable chair with a backrest and a headrest. The angle of the hip joint in the sitting position was set such that it ranged from 70 to 80 degrees of flexion, the knee was set at 70-80 degrees of flexion, and the ankle was maintained at 10 degrees of plantar flexion using a rigid ankle brace. Electromyography (EMG) was performed using Ag/AgCl-plated surface electrodes (diameter $1 \mathrm{~cm}$ ) placed $2 \mathrm{~cm}$ apart over the tested muscles in the right lower limb. EMG data were obtained from the soleus (SOL, for RI and TMS tests) and tibialis anterior (TA, for TMS tests) muscles. The EMG data were amplified and band-pass filtered $(3 \mathrm{~Hz}$ to $2 \mathrm{kHz}$ ) using Digitimer D360 amplifiers (Digitimer Ltd., Welwyn Garden City, Hertfordshire, United Kingdom). Signals were recorded at a sampling rate of $5 \mathrm{kHz}$ using a Power 1401 data acquisition interface (Cambridge Electronic Design Ltd., Cambridge, United Kingdom), and stored on the computer for subsequent analysis using Signal software (Cambridge Electronic Design Ltd., Cambridge, United Kingdom). The EMG activity was monitored online. If the muscle was not fully relaxed, the trial was rejected and performed over again.

\section{Intermittent Theta Burst Stimulation}

The iTBS protocol consisted of 10 bursts, each composed of three stimuli at $50 \mathrm{~Hz}$ repeated at a theta frequency of $5 \mathrm{~Hz}$ every $10 \mathrm{~s}$, for a total of 600 stimuli (190 s) (Huang et al., 2005). TMS was performed using a MagPro X100 stimulator [Medtronic and (currently) MagVenture A/S, Denmark] to deliver biphasic TMS 
pulses through a figure-eight coil with a diameter of $70 \mathrm{~mm}$ (MC-B70). The stimulating coil was placed over the leg area of the primary motor cortex that was optimal for eliciting responses in the right TA muscle, and oriented such that the current in the brain flowed in a posterior to anterior direction through this site. The stimulation intensity was $80 \%$ of the active motor threshold (AMT). The AMT was defined as the minimum stimulation intensity required to evoke a liminal motor potential in the TA (greater than $200 \mu \mathrm{V}$ in $50 \%$ of the 10 trials) while inducing isometric contraction with EMG amplitudes of $100 \mu \mathrm{V}$ in the TA. For the sham stimulation, iTBS was delivered at $60 \%$ of the AMT with the coil turned over to further reduce the current intensity on the leg area of the primary motor cortex, further reducing the intensity to the cortex (Huang et al., 2009).

\section{Patterned Electrical Stimulation}

We applied electrical stimulation to the common peroneal nerve (CPN) on the right leg using a train of $10100-\mathrm{Hz}$ pulses $(1 \mathrm{~ms}$ pulse width) every $2 \mathrm{~s}$ for $20 \mathrm{~min}$, using an intensity that evoked a $100 \mu \mathrm{V}$ M wave response in the TA at rest, without producing movement of the foot (Perez et al., 2003).

\section{Experimental Paradigm}

The study employed a single-masked, sham-controlled, crossover design. All participants received iTBS and PES during three separate sessions on different days: (1) iTBS-before-PES, (2) iTBS-after-PES, and (3) sham iTBS-before-PES (Figure 1). A computer-generated list randomly assigned the order of the three sessions. The RI and motor cortex excitability (measured by the MEP amplitude) were assessed at baseline (before any stimulation); before PES (Pre-PES); and at 0 (Post0), 15 (Post15), 30 (Post30), and $45 \mathrm{~min}$ (Post45) after PES. At each time point, RI was assessed first, then motor cortex excitability. The three sessions were performed at the same time of day for each participant. The participants were instructed to avoid caffeine and any medication that may have affected the central nervous system before the tests. To prevent carry-over effects from previous interventions, washout intervals of 3 days or more were inserted between sessions.

\section{Reciprocal Inhibition}

Reciprocal inhibition from the TA to SOL muscle was assessed using the SOL H-reflex conditioning-test paradigm. The H-reflex was elicited by stimulating the posterior tibial nerve at the popliteal fossa (1-ms rectangular pulse) with an anode on the patella. Throughout the experiment, the test H-reflex amplitude was maintained at $15-20 \%$ of the amplitude of the maximum motor response for the SOL muscle (Crone et al., 1990). Conditioned stimulation of the $\mathrm{CPN}$ was delivered using surface electrodes positioned below the fibular head. The intensity was calibrated to evoke a $100 \mu \mathrm{V}$ response of the $\mathrm{M}$ wave in the TA at rest. The CPN-stimulating electrode was carefully positioned to avoid activation of the peroneus muscles, thus ensuring a more selective stimulation of the deep peroneal nerve. Electric pulses were supplied by two constant-current stimulators (DS7A; Digitimer, Welwyn, United Kingdom). To confirm optimal disynaptic RI, we checked the H-reflex at an inter-stimulus interval (ISI) of 0,1 , and $2 \mathrm{~ms}$ at the beginning of each session. The ISI for the conditioning test was set at 2, 20, or $100 \mathrm{~ms}$ to trigger inhibition through separate mechanisms (Mizuno et al., 1971). Inhibition at an ISI of $2 \mathrm{~ms}$ is called disynaptic $\mathrm{RI}\left(\mathrm{RI}_{2 \mathrm{~ms}}\right)$ and is mediated by a spinal glycinergic disynaptic inhibitory pathway (Curtis, 1959; Tanaka, 1974). Inhibition at an ISI of $20 \mathrm{~ms}\left(\mathrm{RI}_{20 \mathrm{~ms}}\right)$ is called shortlatency presynaptic inhibition, which is thought to result from presynaptic Ia inhibition of afferent fibers that mediate the H-reflex (Mizuno et al., 1971). Inhibition at an ISI of $100 \mathrm{~ms}$ $\left(\mathrm{RI}_{100 \mathrm{~ms}}\right)$ is called long-latency presynaptic inhibition (Mizuno et al., 1971; Huang et al., 2009). The origin of this effect is less clear and may be attributed to presynaptic inhibition that is modulated through long-loop inhibitory connections beyond the spinal cord (Mizuno et al., 1971; Huang et al., 2009). Stimuli were administered every $8 \mathrm{~s}$ in a random order, for a total of eight trials per condition. The amount of RI (\%) at each ISI was defined as: $\frac{\text { mean test } \mathrm{H}-\text { reflex amplitude-mean conditioned } \mathrm{H} \text {-reflex amplitude }}{\text { mean test } \mathrm{H} \text {-reflex amplitude }} \times 100$.

\section{Motor Cortex Excitability}

To assess changes in motor cortex excitability, we applied singlepulse TMS to the leg area of the primary motor cortex using a double-cone coil connected to a Magstim BiStim2 machine (Magstim Company, Whitland, United Kingdom). The hotspot of the primary motor cortex was confirmed based on induction of the largest MEP amplitude in the TA at rest. The stimulation intensity was adjusted to $120 \%$ of the resting motor threshold (RMT). The RMT was defined as the intensity at which 5 out of 10 stimuli generated a $100 \mu \mathrm{V}$ MEP response in the TA at rest, and was measured at the beginning of each session to set the stimulus intensity for the TMS measurement. Ten stimuli were delivered every $5 \pm 0.5 \mathrm{~s}$ at each time point (Cacchio et al., 2009).

\section{Statistical Analysis}

The peak-to-peak amplitude of each H-reflex response or MEP was measured, and the mean amplitude was automatically calculated using custom-written software (NuCursor; Sobell Department, Institute of Neurology, University College, London, United Kingdom). Changes in the amount of RI were calculated by subtracting data obtained at each testing time point (PrePES, Post0, Post15, Post30, and Post45) from the baseline data. MEP amplitudes were normalized to the baseline amplitude (\%) for statistical analysis. To confirm the relaxation of muscles, we calculated the root mean square (RMS) values of the background EMG activity over a $50 \mathrm{~ms}$ period before the stimuli for the H-reflex and TMS were delivered.

The Shapiro-Wilk test was used to determine whether the RI, MEP, RMT, and AMT data were normally distributed. To compare baseline data between protocols, we used oneway analysis of variance (ANOVA; for normally distributed data) or Kruskal-Wallis tests (for non-normally distributed data) on the non-normalized values of the RI, MEP, RMT, and AMT. A two-way repeated-measures ANOVA was used to assess the effects of the timing of iTBS on normally distributed data for each protocol (iTBS-before-PES, iTBSafter-PES, and sham iTBS-before-PES) and for each testing 

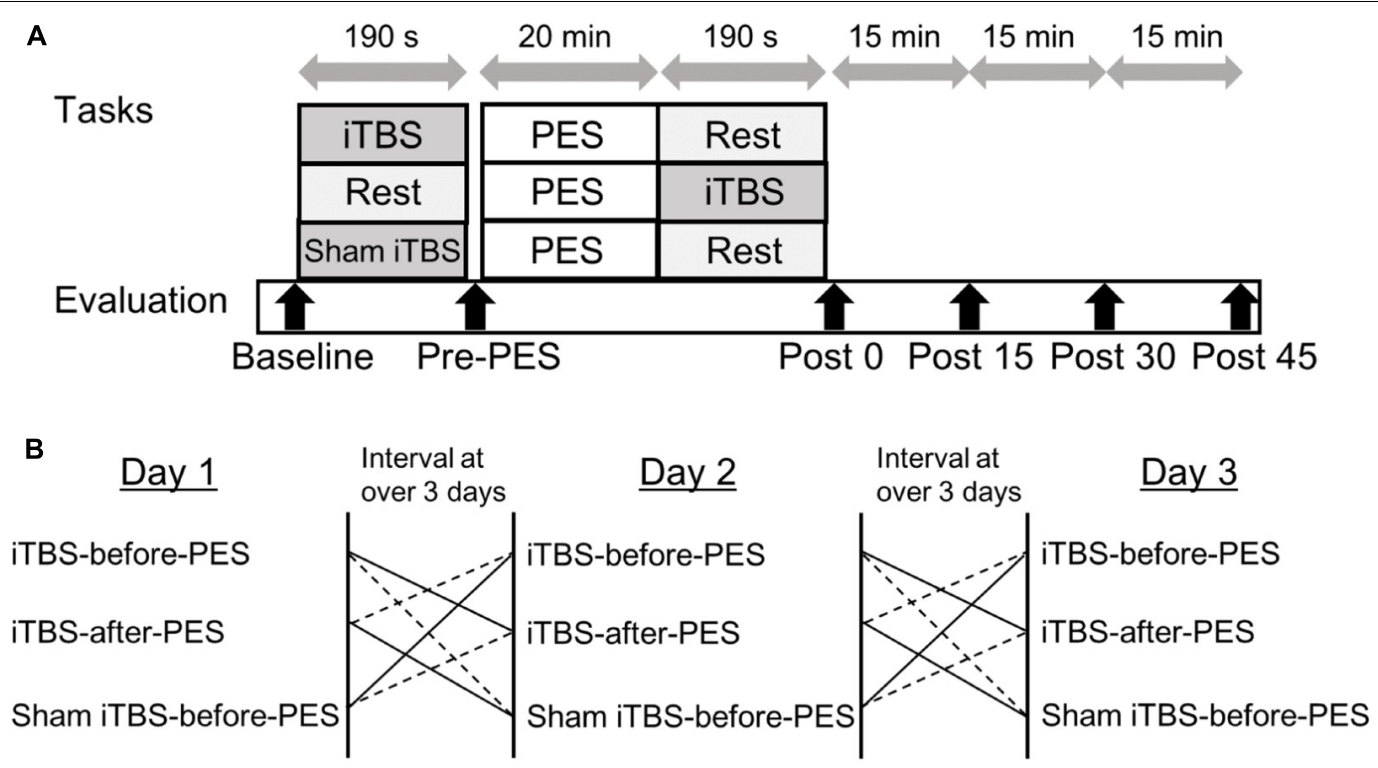

FIGURE 1 | Experimental paradigm for intermittent theta burst stimulation (iTBS) before and after patterned electrical stimulation (PES). (A) Time course of the experiment. (B) Sequence of interventions.

time (Pre-PES, Post0, Post15, Post30, and Post45). Paired $t$-tests with Bonferroni adjustments for multiple comparisons were performed for post hoc comparisons. For the data that were not normally distributed, Mann-Whitney $U$-tests with Bonferroni adjustments were performed to evaluate betweengroup differences. To investigate the relationship between changes in the amount of RI and changes in motor cortex excitability, we performed Pearson correlation analyses (for normally distributed data) or Spearman rank correlation analyses (for non-normally distributed data) between the amount of RI at Post0 and the MEP amplitude at Pre-PES. Results with $P$-values $<0.05$ were considered statistically significant for all analyses. Statistical analyses were performed using IBM SPSS 23.0 (IBM Corp., New York, NY, United States) for Windows.

\section{RESULTS}

The Shapiro-Wilk test confirmed that all data except the normalized MEP values were normally distributed. Two-way repeated-measures ANOVAs revealed no significant interactions between the protocol and testing time and no main effect of the RMS of the background EMG on any outcome in any of the conditions.

\section{RI}

At baseline, the mean $\pm \mathrm{SD}$ of $\mathrm{RI}_{2 \mathrm{~ms}}$ was $7.7(2.6) \%$ in the iTBSbefore-PES session, 9.5 (3.9)\% in the iTBS-after-PES session, and 10.3 (4.3)\% in the sham iTBS-before-PES session. For $\mathrm{RI}_{20 \mathrm{~ms}}$, the mean \pm SD was 7.8 (5.3)\% for iTBS-before-PES, 7.8 (4.3)\% for iTBS-after-PES, and 9.7 (4.4)\% for sham iTBS-before-PES. For
$\mathrm{RI}_{100 \mathrm{~ms}}$, the mean $\pm \mathrm{SD}$ was $12.4(8.1) \%$ in the iTBS-beforePES session, 11.4 (7.4)\% in the iTBS-after-PES session, and 16.3 (8.6)\% in the sham iTBS-before-PES session. The baseline values of RI were not significantly different among the three protocols $\left(\right.$ ANOVA, $\mathrm{RI}_{2 \mathrm{~ms}}: F_{2,27}=1.33, P=0.281 ; \mathrm{RI}_{20 \mathrm{~ms}}: F_{2,27}=0.56$, $P=0.579$; and $\left.\mathrm{RI}_{100 \mathrm{~ms}}: F_{2,27}=1.02, P=0.375\right)$. When comparing the three phases of RI at baseline, we found a significant main effect $\left(F_{2,87}=6.40, P=0.003\right)$. Post hoc analyses revealed that $\mathrm{RI}_{100 \mathrm{~ms}}$ was stronger than either $\mathrm{RI}_{2 \mathrm{~ms}}(P=0.017)$ or $\mathrm{RI}_{20 \mathrm{~ms}}$ $(P=0.004)$.

The time course and values of the RI measures are shown in Figure 2. Significant interactions were found for $\mathrm{RI}_{2 \mathrm{~ms}}$ $\left(F_{8,72}=2.79, P=0.010\right.$, Figure $\left.2 \mathrm{~A}\right), \mathrm{RI}_{20 \mathrm{~ms}}\left(F_{8,72}=2.35\right.$, $P=0.026$, Figure $2 \mathrm{~B})$, and $\mathrm{RI}_{100 \mathrm{~ms}}\left(F_{8,72}=2.08, P=0.049\right.$, Figure 2C). There were significant main effects of protocol and testing time on $\mathrm{RI}_{2 \mathrm{~ms}}$ (protocol: $F_{1.20,10.82}=16.73, P<0.001$; testing time: $F_{4,36}=24.75, P<0.001$ ), $\mathrm{RI}_{20 \mathrm{~ms}}$ (protocol: $F_{2,18}=8.10, P=0.003$; testing time: $\left.F_{4,36}=6.47, P<0.001\right)$, and $\mathrm{RI}_{100 \mathrm{~ms}}$ (protocol: $F_{2,18}=12.45, P<0.001$; testing time: $\left.F_{4,36}=2.11, P=0.030\right)$.

Compared to the Pre-PES values, iTBS-before-PES significantly increased $\mathrm{RI}_{2 \mathrm{~ms}}$ at Post0 $(P=0.002)$ and Post15 $(P=0.047)$, while iTBS-after-PES and sham iTBS-before-PES increased $\mathrm{RI}_{2 \mathrm{~ms}}$ only at Post $0(P=0.028$ and 0.013 , respectively). These results indicate that iTBS prior to PES enhanced PES-induced plasticity for 15 min or longer.

When the amount of RI was compared between protocols at each testing time point, priming iTBS was found to enhance the modulatory effect of PES on $\mathrm{RI}_{2 \mathrm{~ms}}$ and $\mathrm{RI}_{100 \mathrm{~ms}} \cdot \mathrm{RI}_{2 \mathrm{~ms}}$ was significantly increased by iTBS-before-PES (as compared to iTBSafter-PES and sham iTBS-before-PES) at the Post0 and Post15 time points (Post0: vs. iTBS-after-PES, $P=0.011$; vs. sham iTBS-before-PES, $P=0.030$. Post15: vs. iTBS-after-PES, $P=0.038$; 

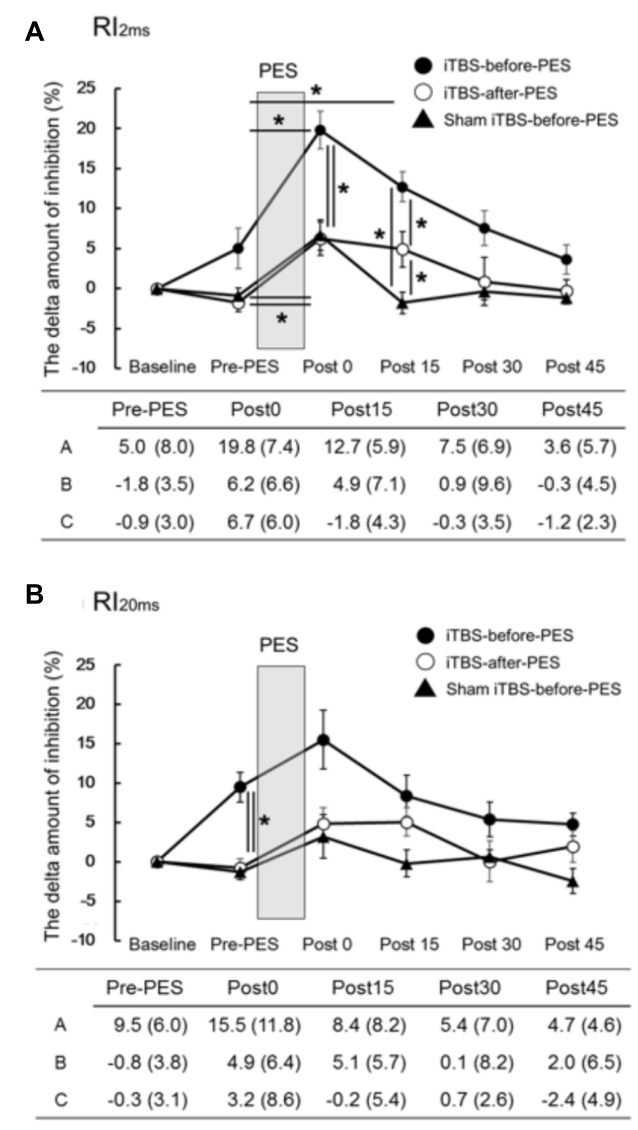

C Rl ${ }_{100 m s}$

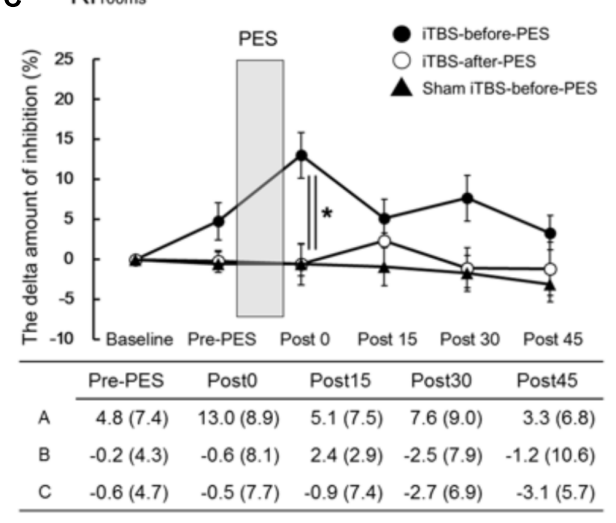

FIGURE 2 | The effects of iTBS before and after PES on RI (A-C). The values (\%) of $\mathrm{Rl}_{2 m s}(\mathbf{A}), \mathrm{Rl}_{20 \mathrm{~ms}}(\mathbf{B})$, and $\mathrm{Rl}_{100 \mathrm{~ms}}$ (C) indicate the delta amount of inhibition, which was calculated by subtracting the values obtained at each testing time point from the baseline values. The values are presented as the mean \pm standard error of the measurements in the figure and as the mean (SD) in the table. In the table, A shows iTBS-before-PES, B shows iTBS-after-PES, and $C$ shows sham iTBS-before-PES. Asterisks indicate significant differences $(P<0.05)$ between Pre-PES and each intervention time point, or within the interventions.

vs. sham iTBS-before-PES, $P<0.001)$. $\mathrm{RI}_{100 \mathrm{~ms}}$ was enhanced only at the Post0 time point (vs. iTBS-after-PES, $P=0.004$; vs. sham iTBS-before-PES, $P=0.007)$. On the other hand,
iTBS-after-PES significantly increased $\mathrm{RI}_{2 \mathrm{~ms}}$ when compared to sham iTBS-before-PES at the Post15 time point $(P=0.017)$.

Furthermore, iTBS-before-PES significantly increased the Pre-PES value of $\mathrm{RI}_{20 \mathrm{~ms}}$ when compared to iTBS-after-PES $(P=0.006)$ and sham iTBS-before-PES $(P=0.002)$. This indicates that iTBS alone can enhance the inhibition measured by $\mathrm{RI}_{20 \mathrm{~ms}}$.

\section{MEPs in the TA and SOL Muscles}

The mean raw values (SD) of the MEP amplitude in the TA muscle at baseline were $0.27(0.17) \mathrm{mV}$ in the iTBS-beforePES session, $0.33(0.24) \mathrm{mV}$ in the iTBS-after-PES session, and $0.31(0.20) \mathrm{mV}$ in the sham iTBS-before-PES session. The baseline values of the MEP amplitude in the TA were not significantly different among the three protocols (Kruskal-Wallis test, $P=0.961$ ). The mean raw values (SD) of the MEP amplitude in the SOL at baseline were $0.24(0.11), 0.32(0.22)$, and 0.30 (0.29) $\mathrm{mV}$, and were not significantly different (Kruskal-Wallis test, $P=0.509)$. The mean values (SD) of RMT/AMT for the TA at baseline were $62(10) \% / 54(11) \%$ of the maximum machine output in the iTBS-before-PES session, 62 (8)\%/55 (12)\% in the iTBS-after-PES session, and $63(10) \% / 57$ (10)\% in the sham iTBSbefore-PES session. There were no significant differences among the three protocols (RMT: ANOVA, $F_{2,27}=0.07, P=0.930$; AMT: ANOVA, $\left.F_{2,27}=0.13, P=0.875\right)$.

The time course and values of the MEP amplitude are shown in Figure 3. Since MEP amplitudes were not normally distributed, non-parametric tests were used to compare them. We used Mann-Whitney $U$-tests with Bonferroni correction to directly compare MEP amplitudes at each time point among protocols, based on the assumption that iTBS increases these amplitudes (Chung et al., 2016; Giboin et al., 2016). In addition, the Spearman rank correlation coefficient was used to analyze the relationship between RI at Post0 and the pre-PES of the MEP amplitude. We found that iTBS-before-PES significantly increased the Pre-PES of the MEP amplitude in the TA muscle compared to iTBS-after-PES $(P=0.004)$ and to sham iTBSbefore-PES ( $P=0.002$; Figure 3A). iTBS-after-PES had no effects on the MEP amplitude at any testing timepoint compared with sham iTBS-before-PES. There were no significant differences among protocols regarding MEP amplitudes in the SOL muscle (Figure 3B).

\section{Correlation Between Changes in Rls and MEP Amplitude in the TA Muscle}

There was a significant positive correlation between $\mathrm{RI}_{2 \mathrm{~ms}}$ at Post 0 and the normalized amplitude of the TA MEP at the PrePES time point ( $r=0.648, P=0.043$, Figure 4A) for iTBS-beforePES. However, no significant correlations were found between $\mathrm{RI}_{20 \mathrm{~ms}}$ and the MEP amplitude in the TA $(r=-0.418, P=0.229$, Figure 4B), or between $\mathrm{RI}_{100 \mathrm{~ms}}$ and the MEP amplitude in the TA ( $r=0.491, P=0.150$, Figure $4 \mathrm{C})$. The results indicate that the enhancement of PES-induced spinal plasticity by iTBS was strongly related to the pre-state changes in cortical excitability modulated by iTBS. 


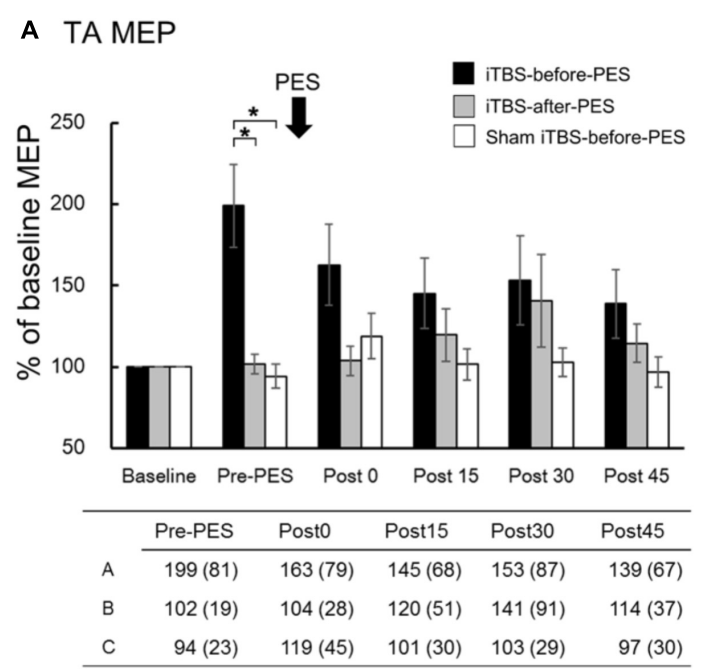

B SOL MEP

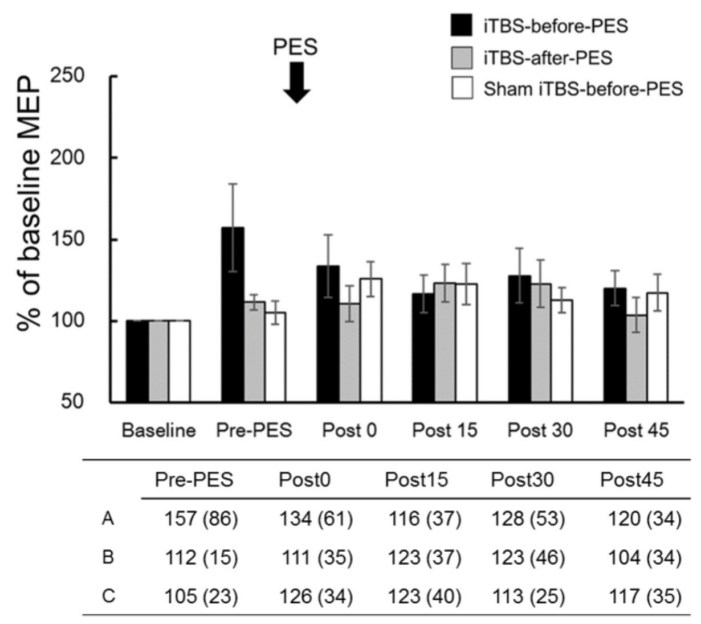

FIGURE 3 | The effects of iTBS before and after PES on motor-evoked potentials (MEPs). MEP amplitudes at the tibialis anterior (TA; $\mathbf{A})$ and soleus (SOL; B) muscles were normalized to the baseline amplitude (\%) for each paradigm. The values (\%) in the figures are presented as the mean \pm standard error. The tables show the mean $( \pm S D)$ for iTBS-before-PES (A),

iTBS-after-PES (B), and sham iTBS-before-PES (C). Asterisks indicate significant differences $(P<0.05)$ among the interventions.

\section{DISCUSSION}

Our results demonstrated that iTBS before, but not after, PES significantly increases and prolongs the PES-induced enhancement of disynaptic RI, and changes the effects of PES on long-loop presynaptic inhibition. The enhancement of disynaptic RI lasts for $15 \mathrm{~min}$ or longer. Our findings provide evidence that spinal plasticity, induced by peripheral sensory input via PES, can be enhanced by priming with iTBS. Moreover, iTBSinduced cortical excitability changes in the TA muscle before PES correlate with changes in disynaptic RI immediately after PES. This indicates that motor cortical excitability changes before PES plays an important role in the induction and maintenance of spinal plasticity.

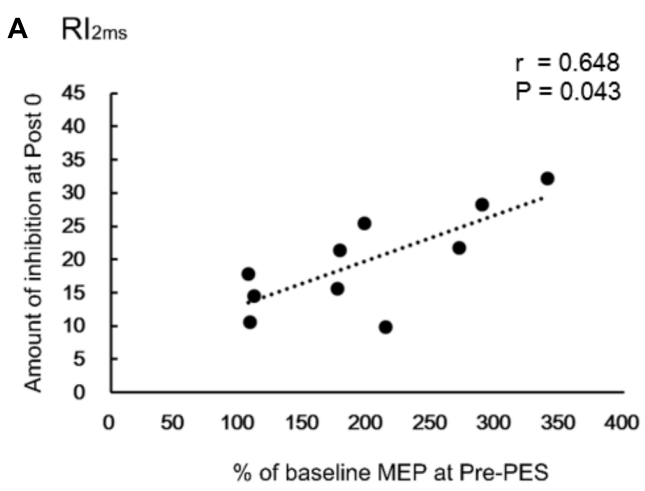

B $\mathrm{Rl}_{20 \mathrm{~ms}}$

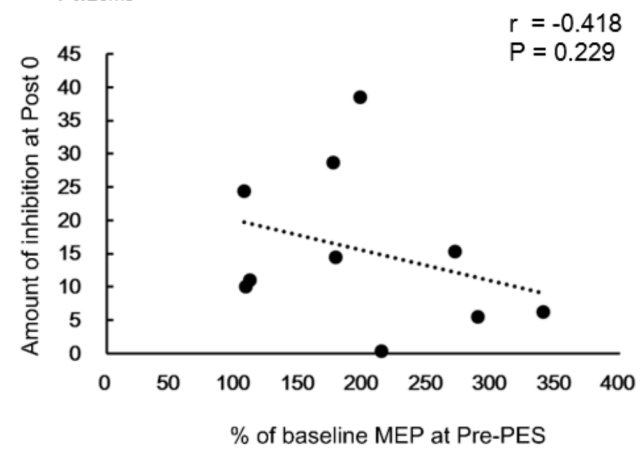

C $\mathrm{Rl}_{100 \mathrm{~ms}}$

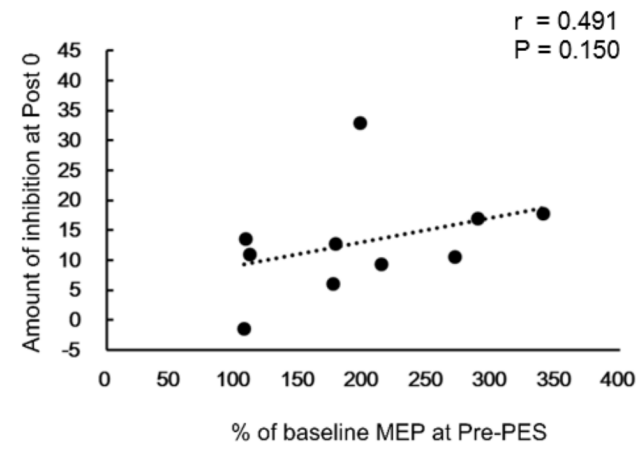

FIGURE 4 | Correlations between change in reciprocal inhibition (RI) and MEPs. Correlation between percent changes in $\mathrm{Rl}_{2 \mathrm{~ms}}(\mathbf{A}), \mathrm{Rl}_{20 \mathrm{~ms}}(\mathbf{B})$, and $\mathrm{Rl}_{100 \mathrm{~ms}}(\mathbf{C})$ at Post0 time-point and the normalized MEPs in the TA muscle, for the iTBS-before-PES condition.

\section{Priming Effects of iTBS on Spinal Plasticity Induced by PES}

Ia inhibitory interneurons, which project to SOL motor neurons, receive convergent inputs from the motor cortex and from the Ia afferents of the TA muscle (Nielsen et al., 1993; Masakado et al., 2001). iTBS is likely to modulate these types of corticospinal projections to spinal inhibitory circuits and thereby enhance the plasticity of disynaptic RI (Figure 5). This finding is supported by previous reports demonstrating that supraspinal modulation plays an important role in the induction and maintenance of spinal plasticity (Chen et al., 2006; Fujiwara et al., 2011; Yamaguchi et al., 2013, 2016; Takahashi et al., 2017). 


\section{Pre-conditioning iTBS}

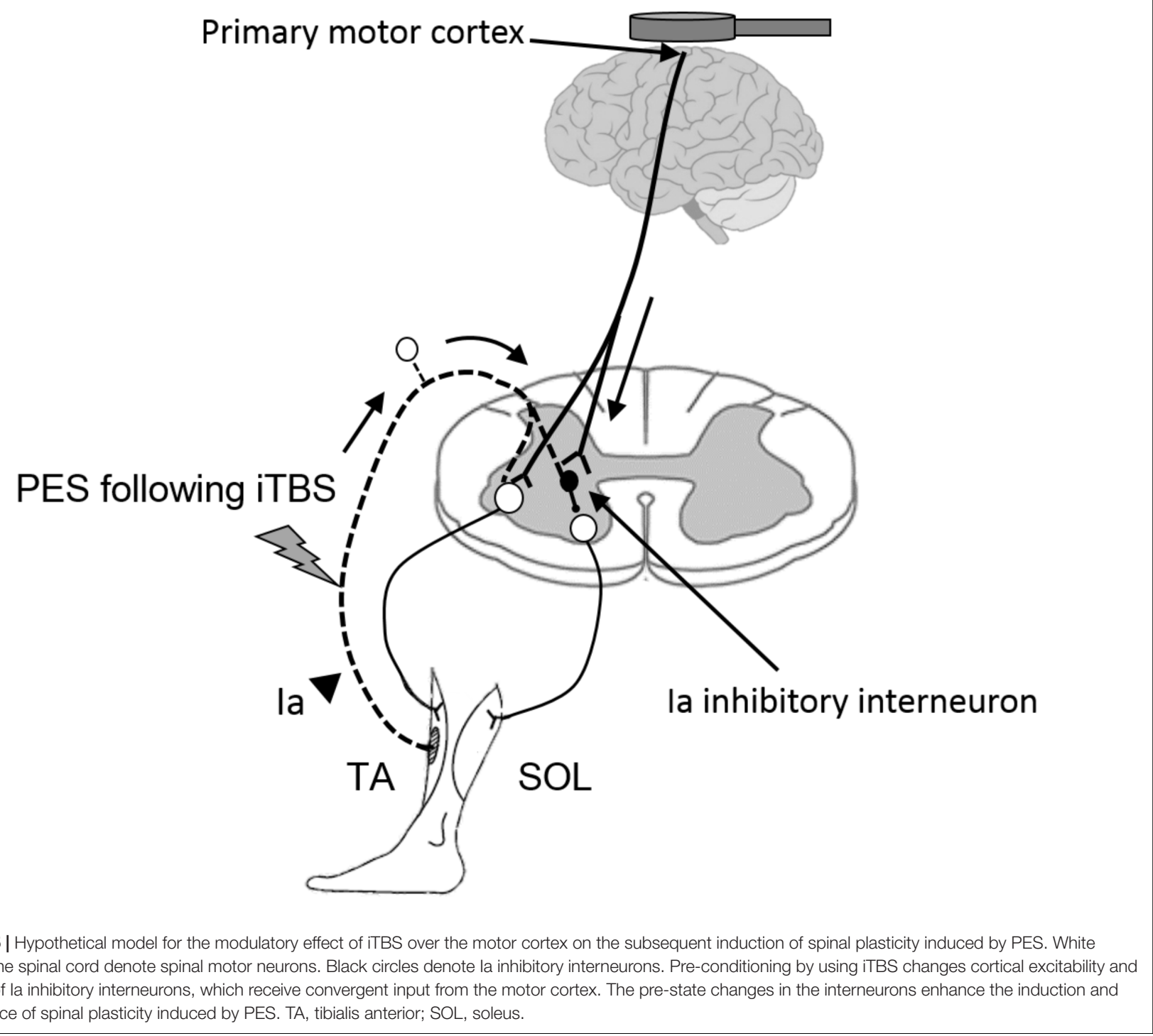

Notably, we observed changes in the plasticity of longlatency presynaptic inhibition with iTBS-before-PES but not with the other two protocols. Accordingly, a previous study showed that the simultaneous application of anodal tDCS over the lower limb motor cortex and peripheral nerve electrical stimulation enhances long-latency presynaptic inhibition (Yamaguchi et al., 2016). However, the physiological mechanism underlying changes in long-latency presynaptic inhibition in these circumstances remains unclear. One possible explanation is that the combination of iTBS and PES modulates primary afferent depolarization (PAD) interneurons that receive convergent input from the motor cortex and from the Ia afferents of the TA muscle (Jankowska et al., 1981; Meunier and Pierrot-Deseilligny, 1998), and that these interneurons mediate long-latency presynaptic inhibition of Ia terminals, which project to SOL motor neurons (Mizuno et al., 1971; Iles and
Pisini, 1992; Pierrot-Deseilligny and Burke, 2012). This finding suggests that the combination of cortical excitability changes and peripheral sensory inputs is a valid strategy to activate PAD interneurons and thereby induce plasticity in long-latency presynaptic inhibition.

The current results highlight the importance of the time sequence of cortical stimulation and sensory inputs for spinal plasticity. Changes in the plasticity of disynaptic RI were stronger for iTBS-before-PES than for iTBS-after-PES, and the effect on long-latency presynaptic inhibition was only seen when iTBS was given before PES. The enhancement of spinal plasticity by priming iTBS may be mediated by heterosynaptic metaplasticity, whereby iTBS-induced changes in corticospinal inputs modulate the subsequent PES-induced plasticity (Holland and Wagner, 1998; Abraham, 2008; Hulme et al., 2014; Parker, 2015). Many studies have reported interactions among the effects 
induced by different non-invasive brain stimulation protocols (Iyer et al., 2003; Ridding and Ziemann, 2010; Müller-Dahlhaus and Ziemann, 2015) through mechanisms of metaplasticity based on the Bienenstock-Cooper-Munro theory of bidirectional synaptic plasticity, which postulates that the threshold for induction of long-term potentiation or long-term depression is dynamically adjusted according to the history of activation in the synapse (Bienenstock et al., 1982). It is known that time is a critical factor affecting metaplastic interactions (Jung and Ziemann, 2009). The current results should promote our understanding of the importance of timing of cortical modulation on spinal plasticity induced by peripheral nerve electrical stimulation.

\section{Relationship Between PES-Induced Spinal Plasticity and Cortical Excitability}

We found that the enhancement of spinal plasticity depended on primed cortical excitability, as altered by iTBS. The increased excitability following iTBS may in turn increase the descending volley from the motor cortex to spinal interneurons and enhance the synaptic strength (Pierrot-Deseilligny and Burke, 2012) of the disynaptic RI circuit. However, we found no correlation between changes in cortical excitability following iTBS and the enhancement of long-latency presynaptic inhibition after PES, nor in short-latency presynaptic inhibition. The simplest explanation for this result is that modulation from the motor cortex to the interneurons mediating short- and long-latency presynaptic inhibitions might be weak compared to the effects on the circuit responsible for disynaptic RI. Indeed, a study found that cortical inhibition evoked by transcranial cortical electrical stimulation clearly correlated with disynaptic RI, but not with short- and long-latency presynaptic inhibition (Iles and Pisini, 1992). Additionally, we showed that baseline inhibition activated by CPN stimulation is larger during long-latency presynaptic inhibition than during disynaptic RI. Previous studies have indicated that the range of long-latency presynaptic inhibition is approximately $20-40 \%$ under resting conditions in healthy individuals, while that of disynaptic RI is approximately $10-20 \%$ (Mizuno et al., 1971; Iles and Pisini, 1992; Fujiwara et al., 2011; Yamaguchi et al., 2013, 2016; Takahashi et al., 2017). Thus, there may be a ceiling effect in detecting the enhancement caused by increased cortical excitability in long-latency presynaptic inhibition.

\section{Effects of iTBS or iTBS Combined With PES on Motor Cortex Excitability and Spinal Inhibition}

In agreement with previous studies on iTBS over the hand and leg area of the primary motor cortex (e.g., Huang et al., 2005; Chung et al., 2016; Giboin et al., 2016), we showed that iTBS facilitates corticospinal excitability of the TA muscle before PES, but does not significantly alter corticospinal excitability of the SOL muscle. The smaller degree of modulation in the SOL may be due to the fact that the intensity of iTBS was set to $80 \%$ of the AMT of the TA. In addition, a figure-eight coil is more focused and more likely to stimulate the TA muscle specifically (Thielscher and Kammer, 2004).

We did not observe a significant effect of iTBS on cortical excitability when we performed iTBS after PES. This may be a consequence of the afferent input stimulated by PES, which might cancel out the effects of the subsequent iTBS on motor cortex excitability. Indeed, previous studies have indicated that afferent stimulation from the CPN suppresses MEPs induced by single-pulse TMS (Roy and Gorassini, 2008; Zewdie et al., 2014). Similarly, the effects of TBS over the motor cortex of the upper limb are known to be modulated or even blocked by muscle activation that occurs around the time of TBS stimulation (Huang et al., 2008; Goldsworthy et al., 2012; Huang, 2016). Furthermore, Rambour et al. (2016) showed that application of iTBS after walking fails to increase motor cortex excitability in the TA muscle. On the other hand, Geertsen et al. (2011) reported that spinal interneuronal pathways modify the descending commands to spinal motor neurons and influence the MEP amplitudes elicited by TMS. Thus, the priming of afferent input stimulated by PES may change the state of synapses and synaptic transmitter release, thereby altering the response to TBS in the motor cortex or at the spinal level. This further confirms that the neuronal states of the brain and even of spinal networks are critical for obtaining optimal effects when using non-invasive brain stimulation (Huang et al., 2017).

\section{Comparison of the Effects of iTBS and tDCS on PES-Induced Plasticity and Spinal Inhibition}

Compared to the 1-mA anodal tDCS used in a previous study (Yamaguchi et al., 2016), iTBS prior to PES may enhance PES-induced spinal plasticity in disynaptic RI and long-latency presynaptic inhibition over a slightly shorter duration (15 vs. $20 \mathrm{~min})$. This is only speculative, as we did not measure the effects of iTBS for more than $20 \mathrm{~min}$ after the PES in the current study. Even though the 1-mA anodal tDCS may lead to longer-lasting enhancement than iTBS, the latter has some advantages, including better spatial and temporal resolution (Thielscher and Kammer, 2004; Huang et al., 2005; Jeffery et al., 2007; Bolognini et al., 2009; McAllister et al., 2009; Madhavan and Stinear, 2010; Roche et al., 2011; Goldsworthy et al., 2012; Tatemoto et al., 2013), which enabled us to study the effects of the temporal relationship of iTBS and PES.

In contrast to previous findings showing that anodal tDCS has no effect on short-latency presynaptic inhibition (Roche et al., 2011; Yamaguchi et al., 2016), we unexpectedly found that iTBS leads to its enhancement. The difference between tDCS and iTBS with respect to short-latency presynaptic inhibition suggests that different mechanisms underlie their after-effects (Di Lazzaro et al., 2010; Roche et al., 2015).

\section{Clinical Implications}

Although the optimal timeframe needed for PES-induced spinal plasticity to improve functional recovery remains unclear, 
enhancement of spinal plasticity is of great importance for the improvement of motor function in patients with incomplete SCI (Bunday and Perez, 2012; Yamaguchi et al., 2016). PES, enhanced by iTBS, may be effective as an adjuvant therapy to other locomotor training therapies, such as treadmill walking with partial body weight support and robot-assisted locomotor training (Smith and Knikou, 2016), while its lasting effects may promote the functional recovery of patients with incomplete SCI.

\section{Limitations}

The sample size of the current study was relatively small. Hence, some marginal results, e.g., the weak correlation between the enhancement of disynaptic RI and increased cortical excitability $(P=0.043)$, should be interpreted cautiously. Another limitation is that our study was conducted in healthy participants. A previous study found that iTBS over the primary motor cortex decreased (rather than increased) the size of MEPs in the arm muscles of patients with SCI (Fassett et al., 2017). Future studies are needed to test the current approach in patients with lower extremity paralysis.

\section{CONCLUSION}

Priming with iTBS enhances and prolongs the modulatory effect of PES on spinal inhibitory circuits, indicating that premodulation of motor cortex excitability plays an important role in spinal plasticity. In contrast, PES prior to iTBS reduces the effect of iTBS on the primary motor cortex, suggesting that afferent inputs may modulate subsequent motor cortex plasticity or the output of motor plasticity at the spinal level. The current findings provide further insight into our understanding of the relationship between the timing of

\section{REFERENCES}

Abraham, W. C. (2008). Metaplasticity: tuning synapses and networks for plasticity. Nat. Rev. Neurosci. 9:387. doi: 10.1038/nrn 2356

Bienenstock, E. L., Cooper, L. N., and Munro, P. W. (1982). Theory for the development of neuron selectivity: orientation specificity and binocular interaction in visual cortex. J. Neurosci. 2, 32-48. doi: 10.1523/JNEUROSCI.0201-00032.1982

Bolognini, N., Pascual-Leone, A., and Fregni, F. (2009). Using non-invasive brain stimulation to augment motor training-induced plasticity. J. Neuroeng. Rehabil. 6:8. doi: 10.1186/1743-0003-6-8

Bunday, K. L., and Perez, M. A. (2012). Motor recovery after spinal cord injury enhanced by strengthening corticospinal synaptic transmission. Curr. Biol. 22, 2355-2361. doi: 10.1016/j.cub.2012.10.046

Cacchio, A., Cimini, N., Alosi, P., Santilli, V., and Marrelli, A. (2009). Reliability of transcranial magnetic stimulation-related measurements of tibialis anterior muscle in healthy subjects. Clin. Neurophysiol. 120, 414-419. doi: 10.1016/j. clinph.2008.11.019

Chen, X. Y., Chen, L., Chen, Y., and Wolpaw, J. R. (2006). Operant conditioning of reciprocal inhibition in rat soleus muscle. J. Neurophysiol. 96, 2144-2150. doi: 10.1152/jn.00253.2006

Chung, S. W., Hill, A. T., Rogasch, N. C., Hoy, K. E., and Fitzgerald, P. B. (2016). Use of theta-burst stimulation in changing excitability of motor cortex: a systematic review and meta-analysis. Neurosci. Biobehav. Rev. 63, 43-64. doi: 10.1016/j.neubiorev.2016.01.008 corticospinal excitability modulation and spinal RI in humans. Further studies are warranted to clarify the clinical application of non-invasive neuromodulation in patients with lower limb paralysis.

\section{AUTHOR CONTRIBUTIONS}

TY, TF, and Y-ZH provided the concept, research design, and project management. TY, TF, ML, and $\mathrm{Y}-\mathrm{ZH}$ wrote the manuscript and procured funding. TY, S-CL, and Y-ZH recruited participants and collected data. TY, S-CL, YT, and KH performed the data analysis. Y-ZH provided facilities and equipment. TY, TF, $\mathrm{KH}, \mathrm{ML}$, and $\mathrm{Y}-\mathrm{ZH}$ provided consultation (including review of manuscript before submission).

\section{FUNDING}

A portion of this study falls under the Japan Agency for Medical Research and Development (AMED) (Grant No. JP18he0402255). This work was partially supported by a JSPS KAKENHI (C) (Grant No. 26350587) and a Health Labor Sciences Research (Grant No. 12102976) to TF, a grant from the Funds for a Grant-in-Aid for Young Scientists (B) (15K16370) and (18K17723) to TY, and grants from the Ministry of Science and Technology, Taiwan (most 105-2314-B-182-004-MY3) and the Chang Gung Medical Foundation (CMRPG3F1251) to Y-ZH.

\section{ACKNOWLEDGMENTS}

The authors would like to thank Dr. Jens Bo Nielsen for his helpful comments.

Crone, C., Hultborn, H., Mazieres, L., Morin, C., Nielsen, J., and PierrotDeseilligny, E. (1990). Sensitivity of monosynaptic test reflexes to facilitation and inhibition as a function of the test reflex size: a study in man and the cat. Exp. Brain Res. 81, 35-45. doi: 10.1007/BF00230098

Curtis, D. R. (1959). Pharmacological investigations upon inhibition of spinal motoneurones. J. Physiol. 145, 175-192. doi: 10.1113/jphysiol.1959.sp0 06134

Di Lazzaro, V., Profice, P., Pilato, F., Dileone, M., Oliviero, A., and Ziemann, U. (2010). The effects of motor cortex rTMS on corticospinal descending activity. Clin. Neurophysiol. 121, 464-473. doi: 10.1016/j.clinph.2009.11.007

Fassett, H. J., Turco, C. V., El-Sayes, J., Lulic, T., Baker, S., Richardson, B., et al. (2017). Transcranial magnetic stimulation with intermittent theta burst stimulation alters corticospinal output in patients with chronic incomplete spinal cord injury. Front. Neurol. 4:380. doi: 10.3389/fneur.2017. 00380

Fujiwara, T., Tsuji, T., Honaga, K., Hase, K., Ushiba, J., and Liu, M. (2011). Transcranial direct current stimulation modulates the spinal plasticity induced with patterned electrical stimulation. Clin. Neurophysiol. 122, 1834-1837. doi: 10.1016/j.clinph.2011.02.002

Geertsen, S. S., van de Ruit, M., Grey, M. J., and Nielsen, J. B. (2011). Spinal inhibition of descending command to soleus motorneurons is removed prior to dorsiflexion. J. Physiol. 589, 5819-5831. doi: 10.1113/jphysiol.2011.214387

Gentner, R., Wankerl, K., Reinsberger, C., Zeller, D., and Classen, J. (2008). Depression of human corticospinal excitability induced by magnetic theta-burst stimulation: evidence of rapid polarity-reversing metaplasticity. Cereb. Cortex 18, 2046-2053. doi: 10.1093/cercor/bhm239 
Giboin, L. S., Thumm, P., Bertschinger, R., and Gruber, M. (2016). Intermittent theta burst over M1 may increase peak power of a wingate anaerobic test and prevent the reduction of voluntary activation measured with transcranial magnetic stimulation. Front. Behav. Neurosci. 10:150. doi: 10.3389/fnbeh.2016. 00150

Goldsworthy, M. R., Pitcher, J. B., and Ridding, M. C. (2012). The application of spaced theta burst protocols induces long-lasting neuroplastic changes in the human motor cortex. Eur. J. Neurosci. 35, 125-134. doi: 10.1111/j.1460-9568. 2011.07924.x

Hinder, M. R., Goss, E. L., Fujiyama, H., Canty, A. J., Garry, M. I., Rodger, J., et al. (2014). Inter- and intra-individual variability following intermittent theta burst stimulation: implications for rehabilitation and recovery. Brain Stimul. 7, 365-371. doi: 10.1016/j.brs.2014.01.004

Holland, L. L., and Wagner, J. J. (1998). Primed facilitation of homosynaptic long-term depression and depotentiation in rat hippocampus. J. Neurosci. 18, 887-894. doi: 10.1523/JNEUROSCI.18-03-00887.1998

Howlett, O. A., Lannin, N. A., Ada, L., and McKinstry, C. (2015). Functional electrical stimulation improves activity after stroke: a systematic review with meta-analysis. Arch. Phys. Med. Rehabil. 96, 934-943. doi: 10.1016/j.apmr.2015. 01.013

Huang, Y. Z. (2016). What do we learn from the influence of motor activities on the after- effect of non-invasive brain stimulation? Clin. Neurophysiol. 127, 1011-1012. doi: 10.1016/j.clinph.2015.11.015

Huang, Y. Z., Edwards, M. J., Rounis, E., Bhatia, K. P., and Rothwell, J. C. (2005). Theta burst stimulation of the human motor cortex. Neuron 45, 201-206. doi: 10.1016/j.neuron.2004.12.033

Huang, Y. Z., Lu, M. K., Antal, A., Classen, J., Nitsche, M., Ziemann, U., et al. (2017). Plasticity induced by non-invasive transcranial brain stimulation: a position paper. Clin. Neurophysiol. 128, 2318-2329. doi: 10.1016/j.clinph.2017. 09.007

Huang, Y. Z., Rothwell, J. C., Edwards, M. J., and Chen, R. S. (2008). Effect of physiological activity on an NMDA-dependent form of cortical plasticity in human. Cereb. Cortex 18, 563-570. doi: 10.1093/cercor/bh m087

Huang, Y. Z., Rothwell, J. C., Lu, C. S., Wang, J., Weng, Y. H., Lai, S. C., et al. (2009). The effect of continuous theta burst stimulation over premotor cortex on circuits in primary motor cortex and spinal cord. Clin. Neurophysiol. 120, 796-801. doi: 10.1016/j.clinph.2009.01.003

Hulme, S. R., Jones, O. D., Raymond, C. R., Sah, P., and Abraham, W. C. (2014). Mechanisms of heterosynaptic metaplasticity. Philos. Trans. R. Soc. Lond. B. Biol. Sci. 369:20130148. doi: 10.1098/rstb.2013.0148

Iles, J. F., and Pisini, J. V. (1992). Cortical modulation of transmission in spinal reflex pathways of man. J. Physiol. 455, 425-446. doi: 10.1113/jphysiol.1992. sp019309

Iyer, M. B., Schleper, N., and Wassermann, E. M. (2003). Priming stimulation enhances the depressant effect of low-frequency repetitive transcranial magnetic stimulation. J. Neurosci. 23, 10867-10872. doi: 10.1523/JNEUROSCI. 23-34-10867.2003

Jankowska, E., McCrea, D., Rudomin, P., and Sykova, E. (1981). Observations on neuronal pathways subserving primary afferent depolarization. J. Neurophysiol. 46, 506-516. doi: 10.1152/jn.1981.46.3.506

Jeffery, D. T., Norton, J. A., Roy, F. F., and Gorassini, M. A. (2007). Effects of transcranial direct current stimulation on the excitability of the leg motor cortex. Exp. Brain Res. 182, 281-287. doi: 10.1007/s00221-007$1093-y$

Jung, P., and Ziemann, U. (2009). Homeostatic and nonhomeostatic modulation of learning in human motor cortex. J. Neurosci. 29, 5597-5604. doi: 10.1523/ JNEUROSCI.0222-09.2009

Madhavan, S., and Stinear, J. W. (2010). Focal and bi-directional modulation of lower limb motor cortex using anodal transcranial direct current stimulation. Brain Stimul. 3:42. doi: 10.1016/j.brs.2009.06.005

Masakado, Y., Muraoka, Y., Tomita, Y., and Chino, N. (2001). The effect of transcranial magnetic stimulation on reciprocal inhibition in the human leg. Electromyogr. Clin. Neurophysiol. 41, 429-432.

McAllister, S. M., Rothwell, J. C., and Ridding, M. C. (2009). Selective modulation of intracortical inhibition by low-intensity theta burst stimulation. Clin. Neurophysiol. 120, 820-826. doi: 10.1016/j.clinph.2009. 02.003
Meunier, S., and Pierrot-Deseilligny, E. (1998). Cortical control of presynaptic inhibition of Ia afferents in humans. Exp. Brain Res. 119, 415-426. doi: 10.1007/ s002210050357

Mizuno, Y., Tanaka, R., and Yanagisawa, N. (1971). Reciprocal group I inhibition on triceps surae motorneurons in man. J. Neurophysiol. 34, 1010-1017. doi: 10.1152/jn.1971.34.6.1010

Müller-Dahlhaus, F., and Ziemann, U. (2015). Metaplasticity in human cortex. Neuroscientist 21, 185-202. doi: 10.1177/1073858414526645

Nielsen, J. B., Petersen, N., Deuschl, G., and Ballegaard, M. (1993). Taskrelated changes in the effect of magnetic brain stimulation on spinal neurones in man. J. Physiol. 471, 223-243. doi: 10.1113/jphysiol.1993.sp01 9899

Nitsche, M. A., and Paulus, W. (2000). Excitability changes induced in the human motor cortex by weak transcranial direct current stimulation. J. Physiol. 527, 633-639. doi: 10.1111/j.1469-7793.2000.t01-1-00633.x

Parker, D. (2015). Synaptic variability introduces state-dependent modulation of excitatory spinal cord synapses. Neural. Plast. 2015, 512156. doi: 10.1155/2015/ 512156

Perez, M. A., Field-Fote, E. C., and Floeter, M. K. (2003). Patterned sensory stimulation induces plasticity in reciprocal Ia inhibition in humans. J. Neurosci. 23, 2014-2018. doi: 10.1523/JNEUROSCI.23-06-02014. 2003

Pierrot-Deseilligny, E., and Burke, D. (2012). The Circuitry of the Human Spinal Cord: Spinal and Corticospinal Mechanisms of Movement. Cambridge: Cambridge University Press. doi: 10.1017/CBO9781139026727

Rambour, M., Caux-Dedeystère, A., Devanne, H., Defebvre, L., Derambure, P., and Delval, A. (2016). Influence of repetitive transcranial magnetic stimulation on tibialis anterior activity during walking in humans. Neurosci. Lett. 11, 49-56. doi: 10.1016/j.neulet.2016.01.027

Ridding, M. C., and Ziemann, U. (2010). Determinants of the induction of cortical plasticity by non-invasive brain stimulation in healthy subjects. J. Physiol. 588, 2291-2304. doi: 10.1113/jphysiol.2010.190314

Roche, N., Geiger, M., and Bussel, B. (2015). Mechanisms underlying transcranial direct current stimulation in rehabilitation. Ann. Phys. Rehabil. Med. 58, 214-219. doi: 10.1016/j.rehab.2015.04.009

Roche, N., Lackmy, A., Achache, V., Bussel, B., and Katz, R. (2011). Effects of anodal transcranial direct current stimulation over the leg motor area on lumbar spinal network excitability in healthy subjects. J. Physiol. 589, 2813-2826. doi: 10.1113/jphysiol.2011.205161

Roy, F. D., and Gorassini, M. A. (2008). Peripheral sensory activation of cortical circuits in the leg motor cortex of man. J. Physiol. 586, 4091-4105. doi: 10.1113/ jphysiol.2008.153726

Smith, A. C., and Knikou, M. A. (2016). Review on locomotor training after spinal cord injury: reorganization of spinal neuronal circuits and recovery of motor function. Neural. Plast. 2016:1216258. doi: 10.1155/2016/121 6258

Takahashi, Y., Fujiwara, T., Yamaguchi, T., Kawakami, M., Mizuno, K., and Liu, M. (2017). The effects of patterned electrical stimulation combined with voluntary contraction on spinal reciprocal inhibition in healthy individuals. Neuroreport 28, 434-438. doi: 10.1097/WNR.0000000000000777

Tanaka, R. (1974). Reciprocal Ia inhibition during voluntary movements in man. Exp. Brain Res. 21, 529-540. doi: 10.1007/BF00237171

Tatemoto, T., Yamaguchi, T., Yohei, O., Kondo, K., and Tanaka, S. (2013). “Anodal transcranial direct current stimulation over the lower limb motor cortex increases the cortical excitability with extracephalic reference electrodes," in Converging Clinical and Engineering Research on Neurorehabilitation, eds J. L. Pons, D. Torricelli, and M. Pajaro (Berlin: Springer), 829-834.

Thielscher, A., and Kammer, T. (2004). Electric field properties of two commercial figure-8 coils in TMS: calculation of focality and efficiency. Clin. Neurophysiol. 115, 1697-1708. doi: 10.1016/j.clinph.2004.02.019

Wang, R. Y., Tseng, H. Y., Liao, K. K., Wang, C. J., Lai, K. L., and Yang, Y. R. (2012). rTMS combined with task-oriented training to improve symmetry of interhemispheric corticomotor excitability and gait performance after stroke: a randomized trial. Neurorehabil. Neural Repair 26, 222-230. doi: 10.1177/ 1545968311423265

Wolpaw, J. R. (2007). Spinal cord plasticity in acquisition and maintenance of motor skills. Acta Physiol. 189, 155-169. doi: 10.1111/j.1748-1716.2006.01656.x 
Wolpaw, J. R., and Tennissen, A. M. (2001). Activity-dependent spinal cord plasticity in health and disease. Annu. Rev. Neurosci. 24, 807-843. doi: 10.1146/ annurev.neuro.24.1.807

Yamaguchi, T., Fujiwara, T., Saito, K., Tanabe, S., Muraoka, Y., Otaka, Y., et al. (2013). The effect of active pedaling combined with electrical stimulation on spinal reciprocal inhibition. J. Electromyogr. Kinesiol. 23, 190-194. doi: 10.1016/ j.jelekin.2012.08.007

Yamaguchi, T., Fujiwara, T., Tsai, Y. A., Tang, S. C., Kawakami, M., Mizuno, K., et al. (2016). The effects of anodal transcranial direct current stimulation and patterned electrical stimulation on spinal inhibitory interneurons and motor function in patients with spinal cord injury. Exp. Brain Res. 234, 1469-1478. doi: 10.1007/s00221-016-4561-4

Zewdie, E. T., Roy, F. D., Okuma, Y., Yang, J. F., and Gorassini, M. A. (2014). Longlatency, inhibitory spinal pathway to ankle flexors activated by homonymous group 1 afferents. J. Neurophysiol. 111, 2544-2553. doi: 10.1152/jn.00673. 2013

Conflict of Interest Statement: The authors declare that the research was conducted in the absence of any commercial or financial relationships that could be construed as a potential conflict of interest.

Copyright (๑) 2018 Yamaguchi, Fujiwara, Lin, Takahashi, Hatori, Liu and Huang. This is an open-access article distributed under the terms of the Creative Commons Attribution License (CC BY). The use, distribution or reproduction in other forums is permitted, provided the original author(s) and the copyright owner(s) are credited and that the original publication in this journal is cited, in accordance with accepted academic practice. No use, distribution or reproduction is permitted which does not comply with these terms. 